\title{
Uso de concentrados autólogos de plaquetas obtenidos mediante el método del tubo como tratamiento de artropatías en caballos
}

\author{
Use of autologous platelet concentrates obtained by the tube method as \\ a treatment for arthropathies in horses
}

\author{
JU Carmona ${ }^{a^{*},}$ C López ${ }^{\mathrm{a}}$, M Prades $^{\mathrm{b}}$ \\ ${ }^{a}$ Grupo de Investigación Terapia Regenerativa, Departamento de Salud Animal, Universidad de Caldas. \\ Calle 65 N $^{\circ}$ 26-10, Manizales, Caldas, Colombia. \\ bDepartamento de Medicina y Cirugía Animal, Facultad de Veterinaria, Universidad Autónoma de Barcelona. \\ Edificio V, 08193, Cerdanyola del Vallès, Barcelona, España.
}

\begin{abstract}
SUMMARY
The clinical effect of the intra-articular injection of an autologous platelet concentrate (APC) in 7 horses with severe joint disease (4 with osteoarthritis and 3 with osteochondrosis) was evaluated. The degree of lameness (DL) and joint effusion (JE) were recorded. Three injections of the APC were performed at two week intervals. Horses were evaluated before each injection and two months after the last treatment. Clinical follow-up was conducted during 1 year. Count of platelets, leucocytes, and determination of transforming growth factor beta 1 (TGF- $\beta_{1}$ ) levels per ml of the APC were performed. A mean of 250 (range: $140-480) \times 10^{6}$ platelets, 8.68 (3.3-18) leucocytes x $10^{6}$, and 12.5 (3-15) ng of TGF- $\beta_{1}$ per ml of the APC were obtained. No adverse clinical signs resulted from this treatment. Horses treated with APCs showed a clinical improvement in both the DL and JE. The most remarkable improvement was observed 2 months after the last treatment and apparently persisted up until 8 months later. Despite the seemingly positive effects of this substance, the clinical use of APCs cannot be recommended until further studies with higher number of cases and longer follow up can be undertaken.
\end{abstract}

Palabras clave: equino, enfermedad articular degenerativa, plaquetas.

Keywords: equine, degenerative joint disease, platelet.

\section{INTRODUCCIÓN}

Los concentrados autólogos de plaquetas (APCs) son una fuente de diversos factores de crecimiento (GFs), tales como el GF transformador beta (TGF- $\beta$ ), GF insulínico tipo I (IGF-I) y GF derivado de las plaquetas (PDGF), y otras moléculas que modulan la inflamación y reparación tisular (Anitua y col 2004). Los APCs se han empleado en reconstrucción alveolomaxilar, cirugía plástica (Anitua y col 2004), para tratar lesiones tendino-ligamentosas en atletas humanos e incluso se ha documentado un caso de avulsión cartilaginosa no traumática en un futbolista con resolución favorable mediante el uso de APCs (Sánchez y col 2003).

El incremento del uso de APCs ocurre en medicina humana (Sánchez y col 2003, Anitua y col 2004) y no en medicina equina, lo que hace el tema interesante pero no claro y reivindica más investigaciones. La propuesta de este trabajo de investigación clínica se basó en que

\footnotetext{
Aceptado: 11.03.2009.

* Departamento de Salud Animal, Universidad de Caldas, Calle 65 № 26-10, Manizales, Caldas, Colombia; carmona@ucaldas.edu.co
}

los APC equinos son una fuente importante de TGF- $\beta$ (Argüelles y col 2006, Carmona y col 2008), IGF-I y PDGF (Schnabel y col 2007), los cuales tienen efecto benéfico sobre el metabolismo del cartílago articular (Fortier y col 1997, Frisbie y col 2000). Las hipótesis planteadas fueron que la inyección intraarticular de APCs en caballos con artropatía natural podría ser benéfica al reducir los signos clínicos asociados a las mismas, tales como cojera y distensión sinovial y no producir ninguna sintomatología adversa asociada con el tratamiento, tales como fiebre, inflamación local y cojera. Para comprobar esto se analizó el efecto de la inyección intraarticular de APCs obtenidos mediante el método del tubo (Argüelles y col 2006) sobre el grado de cojera, distensión sinovial (articular) en caballos con artropatía natural. Cada paciente tratado fue estrechamente vigilado para reconocer cualquier reacción adversa (sistémica o local, inmediata o retardada), asociada con el tratamiento experimental.

\section{MATERIAL Y MÉTODOS}

Este estudio clínico fue aprobado por la dirección del Hospital Clínico Veterinario de la Universidad Autónoma de Barcelona, España. 
ANIMALES

Siete caballos de diferente raza, sexo y edad fueron incluidos en el estudio. Tres caballos tenían osteoartritis (OA) no relacionada con osteocondrosis (OCD), un caballo tenía OA de la articulación femorotibial medial secundaria a OCD y lesión del menisco medial del mismo lado, dos potrancas tenían OCD bilateral de las babillas y un potro tenía OCD de la articulación tarsocrural izquierda (cuadro 1). Las dos potrancas con OCD habían sufrido artroscopia bilateral con desbridamiento de más del $40 \%$ de los bordes trocleares laterales de ambos fémur. Se decidió incluir esas pacientes ya que después de la cirugía quedaron varios fragmentos osteocondrales articulares y los propietarios no consintieron una segunda cirugía. El potro con OCD tarsocrural izquierda fue incluido porque su propietario no estaba interesado en la cirugía. Los caballos con OA habían sido tratados con descanso durante un periodo de 2 meses a 1 año y habían recibido corticoesteroides y ácido hialurónico vía intraarticular. Una de las potrancas había sido operada tres meses antes del tratamiento y la otra potranca seis meses antes.

\section{CRITERIOS DE INCLUSIÓN}

El criterio de inclusión comprendió un examen musculoesquelético detallado y procedimientos diagnósticos auxiliares, como radiografía, ecografía y bloqueos anestésicos regionales. Sólo se incluyeron caballos que presentaran signos clínicos de artropatía (e.j.: pérdida progresiva de función articular, dolor, engrosamiento de la cápsula articular y distensión sinovial (articular)) con al menos un año de duración y signos radiográficos de $\mathrm{OA}$ primaria o secundaria a $\mathrm{OCD}$ o con fragmentos osteocondrales. Los signos radiológicos incluían remodelación del hueso subcondral, osteofitosis marginal y presencia de fragmentos osteocondrales (en los casos de OA secundaria a OCD). Sólo se incluyeron pacientes que no habían recibido ningún tratamiento médico con antiinflamatorios no esteroidales, corticoesteroides intraarticulares, hialuronato, glicosaminoglicanos u otras preparaciones antiartríticas, al menos dos meses antes de la inclusión en el estudio y un año durante el mismo.

\section{ESQUEMA DEL TRATAMIENTO}

Cada paciente fue tratado con una inyección intraarticular de un APC obtenido mediante el método del tubo (Argüelles y col 2006, Carmona y col 2008) cada 15 días, por tres veces consecutivas.

\section{DISEÑO DEL ESTUDIO CLÍNICO}

Cada paciente fue evaluado para grado de cojera y grado de distensión (efusión) articular al inicio del estudio, antes de cada inyección de APC, dos meses después del último tratamiento y fueron seguidos clínicamente hasta un año después de finalizar el tratamiento.

Determinación del grado de cojera. El grado de cojera fue determinado por observación de los pacientes cuando trotaban en línea recta sobre superficie dura. La calificación del grado de cojera fue efectuada según los criterios de la AAEP (1991) de 0-5.

Determinación del grado de distensión articular. El grado de distensión articular fue construido al combinar

Cuadro 1. Reseña e historia clínica de los pacientes del estudio. Signalment and clinical signs of the horses of the study.

\begin{tabular}{|c|c|c|c|c|c|c|c|c|}
\hline $\begin{array}{l}\text { Caballo } \\
\text { No }\end{array}$ & Raza & Sexo & $\begin{array}{l}\text { Edad } \\
\text { (Años) }\end{array}$ & Disciplina & $\begin{array}{l}\text { Articulación } \\
\text { afectada }\end{array}$ & Diagnóstico & $\begin{array}{l}\text { Tratamiento } \\
\text { Previo }\end{array}$ & $\begin{array}{l}\text { APC } \\
\text { (ml/art) }\end{array}$ \\
\hline 1 & Árabe & Macho & 7 & Enduro & $\begin{array}{l}\text { Ambos menudillos } \\
\text { anteriores }\end{array}$ & OA & Esteroides IA & 10 \\
\hline 2 & Haflinger & Macho & 15 & Doma clásica & $\begin{array}{l}\text { Interfalangeana distal } \\
\text { anterior izquierda }\end{array}$ & OA & $\begin{array}{l}\text { Hialuronato y } \\
\text { esteroides IA }\end{array}$ & 10 \\
\hline 3 & $\begin{array}{l}\text { Caballo Francés } \\
\text { de Montaña }\end{array}$ & Hembra & 8 & Tiro & $\begin{array}{l}\text { Tibiotarsiana, proximal e } \\
\text { intertarsiana izquierdas }\end{array}$ & $\mathrm{OA}$ & NSAIDs & 15 \\
\hline 4 & Warmblood & Macho & 6 & Salto & $\begin{array}{l}\text { femorotibial medial } \\
\text { derecha con lesión del } \\
\text { menisco medial }\end{array}$ & $\mathrm{OCD} / \mathrm{OA}$ & NSAIDs & 20 \\
\hline 5 & Hannoveriana & Potra & 1,4 & Salto (uso potencial) & Babillas & OCD & $\begin{array}{l}\text { Artroscopia, } \\
\text { hialuronato y } \\
\text { esteroides IA }\end{array}$ & 20 \\
\hline 6 & $\mathrm{CDE}$ & Potra & 1,2 & Salto (uso potencial) & Babillas & OCD & Artroscopia & 20 \\
\hline 7 & Holsteiner & Macho & 3,5 & Salto & Tibiotarsiana izquierda & OCD & $\begin{array}{l}\text { Ninguna terapia } \\
\text { previa }\end{array}$ & 15 \\
\hline
\end{tabular}

APC: Concentrado autólogo de plaquetas. CDE: Caballo de deporte español. IA: Intraarticular. NSAIDs: Antiinflamatorios no esteroidales. OCD: Osteocondrosis. OA: Osteoartritis. 
los hallazgos clínicos y ecográficos de las articulaciones afectadas. Mediante ecografía se evaluaron la superficie articular, la cantidad de fluido sinovial, el espesor y apariencia de la membrana sinovial y sus vellos sinoviales. La evaluación ecográfica siempre fue determinada en el mismo sitio anatómico, para lo cual se señaló un sitio específico (área de tricotomía) de cada articulación. La valoración clínica incluyó la evaluación de la apariencia externa de la articulación afectada en comparación con la contralateral sana (cuando era posible), temperatura, textura de la cápsula articular, rango de movilidad y grado de dolor durante la palpación y al producir movimiento articular pasivo. El grado de efusión articular fue clasificado de 0 a 3 , donde $0=$ articulación de apariencia normal. 1 = efusión articular ligeramente palpable, sin engrosamiento de la cápsula articular. 2 = efusión articular palpable, cápsula articular moderadamente engrosada y ligera reacción dolorosa durante la palpación articular profunda. 3 = efusión articular evidente, engrosamiento marcado de la membrana sinovial acompañado de signos clínicos de inflamación y con reacción dolorosa acentuada durante la palpación superficial de la cápsula articular y la movilización pasiva de la articulación.

Determinación de los efectos adversos asociados con el tratamiento. Se llevó un registro de reacciones sistémicas y locales adversas postratamiento, durante una semana. La temperatura rectal fue tomada cada 2 horas, durante las primeras 48 horas y cada 6 horas, desde ese momento hasta el día 7. Se tuvo en cuenta el estado de ánimo y la ingesta de cada paciente. Se observó el apoyo de los miembros tratados de los caballos y la aparición de los signos de la inflamación (dolor, calor, rubor, edema) en las articulaciones tratadas.

\section{PREPARACIÓN E INYECCIÓN DEL CONCENTRADO AUTÓLOGO DE PLAQUETAS}

Previa preparación aséptica, se obtuvo sangre entera de la vena yugular de cada paciente, mediante un catéter mariposa 23G (Terumo, Bélgica). La sangre fue depositada en tubos de citrato de sodio $3,2 \% \mathrm{v} / \mathrm{v}$ con capacidad para $5 \mathrm{ml}$. Los tubos con sangre fueron centrifugados a $120 \mathrm{~g}$ durante 5 minutos. La primera fracción supernadante (50\%) del plasma, adyacente a la capa leucoplaquetaria (buffy coat), fue obtenida en una cámara de flujo laminar, depositada en tubos sin aditivo y centrifugada a $240 \mathrm{~g}$ durante 5 minutos. Luego se extrajo el $25 \%$ del plasma del fondo de cada tubo. Esa última fracción fue depositada dentro de jeringas estériles y activada con cloruro de calcio $(4.5 \mathrm{mEq} / 5 \mathrm{ml})$, a razón de $50 \mu \mathrm{l}$ por ml de APC (Argüelles y col 2006, Carmona y col 2008). Dos ml de APC de cada paciente fueron empleados para realizar recuento de plaquetas y leucocitos (ADVIA 120, Bayer, USA) y determinar los niveles de TGF- $\beta_{1}$ mediante una prueba comercial humana de ELISA (R\&D System, USA).

$\mathrm{La}(\mathrm{s})$ articulación(es) afectada(s) de cada paciente fue(ron) preparada(s) asépticamente para realizar la inyección del APC. Los pacientes fueron sedados con un bolo intravenoso de detomidina ( $40 \mu \mathrm{g} / \mathrm{kg} / \mathrm{IV}$ ) (Domosedan, Pfizer, España) y tartrato de butorfanol (0.02 mg/kg/IV) (Torbugesic, Fort Dodge Laboratories Inc, España). La cantidad de APC usado en cada paciente fue determinada en función del tamaño de la articulación afectada (cuadro 1). Se recomendó que los caballos fueran confinados y que dos semanas después de la última inyección se permitiera un aumento gradual del ejercicio.

\section{ANÁLISIS ESTADÍSTICO}

Las valores de grado de cojera, grado de distensión articular y los datos hematológicos (análisis del APC) fueron expresados como medianas con sus respectivos rangos.

\section{RESULTADOS Y DISCUSIÓN}

Se obtuvo un promedio ( $n: 7)$ de 250 (rango: 140480) x $10^{6}$ plaquetas, $8,68(3,3-18)$ leucocitos x $10^{6}$, y 12,5 (3-15) ng de TGF- $\beta_{1}$ por ml de APC. La administración intraarticular de los APCs redujo el grado de cojera a partir de la tercera inyección. La mejoría del grado de cojera fue gradual y más evidente después del tercer tratamiento y dos meses después (cuadro 2). No se observaron signos clínicos (sistémicos o locales, inmediatos o retardados) adversos severos como resultado del tratamiento. Sin embargo, una potranca con OCD y un caballo con OA presentaron efusión sinovial moderada y transitoria que desapareció a las 48 h sin ningún tratamiento. Los APCs redujeron el grado de distensión articular. Este efecto fue

Cuadro 2. Evolución del grado de cojera y del grado de distensión articular en los caballos del estudio ( $n$ : 7).

Evolution of the lameness degree and joint distension scores in the horses of the study ( $n: 7)$.

\begin{tabular}{lcccc}
\hline \multirow{2}{*}{ Parámetro } & \multicolumn{4}{c}{ Valor } \\
\cline { 2 - 6 } & Registro inicial & Antes de la 2a inyección & Antes de la 3a inyección & Evaluación final \\
\hline Grado de cojera & 1,1 Rango 0,5-2 & 0,8 Rango 0-1,5 & 0,6 Rango 0-1,5 & 0,35 Rango 0-1 \\
Grado de distensión articular & 2,25 Rango 2-3 & 1,75 Rango 1-2,5 & 1,5 Rango 1-2,5 & 0,875 Rango 0-1 \\
\hline
\end{tabular}


más notable que el de disminución del grado de cojera y más evidente dos meses después del último tratamiento (cuadro 2). Los caballos con OA mantuvieron su último registro de cojera y distensión articular durante aproximadamente 8 meses después de la última inyección del APC y luego mostraron un aumento gradual del mismo. Las dos potrancas y el potro con OCD permanecen sin cojera y efusión sinovial, pero ellos aún no habían sido sometidos a un alto nivel de entrenamiento. Durante el tratamiento los tres pacientes con OCD fueron mantenidos en reposo; la yegua con OA del tarso continuó con su entrenamiento de tiro, pero a baja intensidad; dos caballos con OA fueron ejercitados ligeramente y un caballo con OA bilateral de ambos menudillos fue mantenido en el mismo nivel de exigencia a pesar de las recomendaciones de reducir la carga de trabajo durante el periodo del tratamiento.

La técnica empleada para preparar los APCs de este estudio produjo una baja concentración de plaquetas ( $250 \times 10^{6}$ plaquetas $/ \mathrm{ml}$ ) en comparación con los valores obtenidos mediante el método de aféresis descrito por Carter y col (2003) (490 x $10^{6}$ plaquetas $\left./ \mathrm{ml}\right)$ y por Sutter y col (2004) $\left(855 \times 10^{6}\right.$ plaquetas $\left./ \mathrm{ml}\right)$ o mediante un método semiautomatizado de buffy coat $\left(1472 \times 10^{6}\right.$ plaquetas $/ \mathrm{ml})$. Sin embargo, la técnica de este estudio produjo concentraciones elevadas de TGF- $\beta_{1}(12,5 \mathrm{ng} / \mathrm{ml})$ en comparación con las concentraciones obtenidas mediante aféresis (Carter y col 2003) $(7,5 \mathrm{ng} / \mathrm{ml})$, y bajas de TGF- $\beta_{1}$ en comparación con los resultados descritos por Sutter y col (2003) $(23,6 \mathrm{ng} / \mathrm{ml})$. Sin embargo, es importante aclarar que concentraciones tan bajas como $5 \mathrm{ng} / \mathrm{ml}$ de TGF- $\beta_{1}$ inducen la síntesis de matriz extracelular y la proliferación de condrocitos in vitro (Fortier y col 1997).

La mejoría del grado de cojera y distensión articular observada en los pacientes tratados con APCs se podría explicar por el efecto que ejercen algunos de los GFs plaquetarios sobre la inflamación y reparación tisular (van Miert 2002). En las artropatías como la OA o la OCD la interleucina 1 (IL-1), a través de la interacción con receptores celulares, promueve la expresión de factores nucleares, como el kapa-beta (NF- $\kappa B$ ). Ese factor facilita la expresión de genes que codifican péptidos proinflamatorios secundarios (ej.: IL-6, IL-8, IL-12, etc), quimiocinas, prostanoides, metaloproteinasas y óxido nítrico (Takafuji y col 2002, van Miert 2002) que en el espacio articular producen dolor (cojera) y efusión articular (Carmona y Giraldo-Murillo 2007). Es posible que el APC haya ejercido un efecto analgésico y antiinflamatorio al inhibir la expresión de factores nucleares o bloquear la acción de metabolitos proinflamatorios en una escala inferior. Los APCs son una fuente importante de TGF- $\beta$ s (Argüelles y col 2006, Carmona y col 2008). La expresión de esos péptidos son claves para disminuir el dolor y la inflamación articular y la progresión del deterioro del cartílago articular (Marinova-Mutafchieva y col 2006). Por otra parte, se ha demostrado in vitro que los APCs aumentan la síntesis de ácido hialurónico en sinoviocitos de humanos con OA (Anitua y col 2007). El ácido hialurónico posee un poderoso y prolongado efecto analgésico y antiinflamatorio a nivel articular, posiblemente mediado por el recubrimiento físico de los receptores de las diferentes células articulares, especialmente las inflamatorias (Carmona y Giraldo-Murillo 2007). Infortunadamente, estos metabolitos no fueron medidos en el líquido sinovial de los caballos tratados con los APCs.

El efecto terapéutico, pero temporal y limitado, de los fármacos convencionales empleados para el tratamiento de las artropatías, es un problema importante en clínica equina (Carmona y Giraldo-Murillo 2007). Los caballos con OA deben ser tratados periódicamente. Pocos fármacos pueden modificar el curso o progresión de la enfermedad e incluso muchos aceleran la destrucción articular (Carmona y Giraldo-Murillo 2007). La suma de los hallazgos terapéuticos del APC (efectos analgésico y antiinflamatorio prolongados), observados en esta muestra de caballos hace pensar que este es un tratamiento inductor de remisión (Malemud y col 2003). Sin embargo, los efectos de los APCs deberían ser evaluados de una manera más detallada, tanto en sistemas de experimentación in vitro como en modelos in vivo, para poder esclarecer su verdadero efecto clínico y molecular al respecto.

Finalmente, con los resultados de este pequeño estudio no se puede recomendar plenamente la inyección intraarticular de equinos con artropatía natural. Sin embargo, se puede concluir que la inyección articular de los APCs puede ser considerada con un procedimiento seguro, siempre y cuando se conserven estrictas medidas de asepsia, tanto en la preparación (cámara de flujo laminar) como en la inyección articular de la sustancia.

\section{RESUMEN}

Se evaluó el efecto clínico de la inyección intraarticular de un concentrado autólogo de plaquetas (APC) en 7 caballos con enfermedad articular grave (4 con osteoartritis y 3 con osteocondrosis). Se documentó el grado de cojera (GC) y distensión articular (DA). Se aplicaron tres inyecciones de APCs con un intervalo de dos semanas entre cada aplicación. Los caballos fueron evaluados antes de cada inyección y dos meses después del último tratamiento. Se realizó seguimiento clínico durante un año. Se determinaron los niveles de plaquetas y leucocitos y los niveles de factor de crecimiento transformante beta 1 $\left(\right.$ TGF- $\beta_{1} \cdot$ ) por ml de APC. El APC preparado presentó un promedio de 250 (rango: $140-480)$ x $10^{6}$ plaquetas y 8,68 (3,3-18) x $10^{6}$ leucocitos por y 12,5 (3-15) ng de TGF. $\beta_{1}$. La inyección de los APCs produjo una mejoría clínica en el GC y DA. La mejoría más marcada se observó dos meses después del último tratamiento y persistió durante ocho meses posinyección. No se observaron signos clínicos adversos significativos asociados al tratamiento. A pesar de los efectos aparentemente positivos del APC no se puede recomendar el uso de esa sustancia, hasta que no haya un suficiente número de casos clínicos preferiblemente evaluados mediante un estudio doble ciego controlado.

\section{AGRADECIMIENTOS}

Los autores agradecen a todas las personas de la Unidad Equina del Hospital Clínico Veterinario de la Universidad Autónoma de Barcelona y 
del Instituto de Terapia Regenerativa Tisular, Clínica Teknon, Barcelona, España, que hicieron posible este trabajo de investigación.

\section{REFERENCIAS}

AAEP, American Association of Equine Practitioners. 1991. Guide for veterinary service and judging of equestrian events: definition and classification of lameness. Lexington: AAEP, USA, Pp 19.

Anitua E, I Andía, B Ardanza, P Nurden, AT Nurden. 2004. Autologous platelets as a source of proteins for healing and tissue regeneration. Thromb Haemost 91, 4-15.

Anitua E, M Sánchez, AT Nurden, MM Zalduendo, M de la Fuente, J Azofra, I Andía. 2007. Platelet-released growth factors enhance the secretion of hyaluronic acid and induce hepatocyte growth factor production by synovial fibroblasts from arthritic patients. Rheumatology (Oxford) 46, 1769-1772.

Argüelles D, JU Carmona, J Pastor, A Iborra, L Viñals, P Martinez, E Bach, M Prades. 2006. Evaluation of single and double centrifugation tube method for concentrating equine platelets. Res Vet Sci 81, 237-245.

Carmona JU, CE Giraldo-Murillo. 2007. Fisiopatología y tratamiento convencional de la osteoartritis en el caballo. Vet Zootec 1, 60-73.

Carmona JU, D Argüelles, M Prades. 2008. Niveles de factor de crecimiento transformante beta- 3 y óxido nítrico en cuatro concentrados autólogos de plaquetas y plasma derivados de sangre equina. Arch Med Vet 40, 155-160.

Carter CA, DG Jolly, CE Worden, DG Hendren, CJ Kane. 2003. Plateletrich gel promotes differentiation and regeneration during equine wound healing. Exp Mol Path 74, 244-255.

Fortier LA, AJ Nixon, HO Mohammed, G Lust. 1997. Altered biological activity of equine chondrocytes cultured in a three-dimensional fibrin matrix and supplemented with transforming growth factor beta-1. Am J Vet Res 58, 66-70.

Frisbie DD, EA Sandler, GW Trotter, CW McIlwraith. 2000. Metabolic and mitogenic activities of insulin-like growth factor-1 in interleukin1-conditioned equine cartilage. Am J Vet Res 61, 436-441.

Malemud CJ, N Islam, TM Haqqi. 2003. Pathophysiological mechanisms in osteoarthritis lead to novel therapeutic strategies. Cells Tissues Organs 174, 34-48.

Marinova-Mutafchieva L, C Gabay, K Funa, RO Williams. 2006. Remission of collagen-induced arthritis is associated with high levels of transforming growth factor-beta expression in the joint. Clin Exp Immunol 46, 287-293.

Sánchez M, J Azofra, E Anitua, I Andia, S Padilla, J Santisteban, I Mujika. 2003. Plasma rich in growth factors to treat an articular cartilage avulsion: a case report. Med Scien Sports Exer 35, 1648-1652.

Schnabel LV, HO Mohammed, BJ Millar, WG McDermott, MS Jacobson, KS Santangelo, LA Fortier. 2007. Platelet Rich Plasma (PRP) Enhances Anabolic Gene Expression Patterns in Flexor Digitorum Superficialis Tendons. J Orthop Res 25, 230-240.

Sutter WW, AJ Kaneps, AL Bertone. 2004. Comparison of hematologic values and transforming growth factor- $\beta$ and isulin-like growth factor concentrations in platelets concentrates obtained by use of buffy coat and apheresis methods from equine blood. Am J Vet Res 65, 924-930.

Takafuji VA, CW McIlwraith, RD Howard. 2002. Effects of equine recombinant interleukin-1alpha and interleukin-1beta on proteoglycan metabolism and prostaglandin E2 synthesis in equine articular cartilage explants. Am J Vet Res 63, 551-558.

van Miert ASJ. 2002. Present concepts on the inflammatory modulators with special reference to cytokines. Vet Res Commun 26, 111-126. 